\title{
Genetic identification of pathogenic variations of the DMD gene: a retrospective study from 10,481 neonatal patients based on next-generation sequencing data
}

\author{
Tiantian Xiao ${ }^{1,2 \#}$, Bingbing $\mathrm{Wu}^{3 \#}$, Yun Cao ${ }^{1}$, Renchao Liu ${ }^{3}$, Guoqiang Cheng ${ }^{1}$, Laishuan Wang ${ }^{4}$, \\ Deyi Zhuang ${ }^{5}$, Zhengyan Zhao ${ }^{6}$, Huijun Wang ${ }^{3}$, Wenhao Zhou ${ }^{1,3}$
}

${ }^{1}$ Department of Neonates, Key Laboratory of Neonatal Diseases, Ministry of Health, Children's Hospital of Fudan University, National Children's Medical Center, Shanghai, China; ${ }^{2}$ Department of Neonatology, Chengdu Women's and Children's Central Hospital, School of Medicine, University of Electronic Science and Technology of China, Chengdu, China; ${ }^{3}$ Center for Molecular Medicine, Children's Hospital of Fudan University, National Children's Medical Center, Shanghai, China; ${ }^{4}$ Department of Pediatrics, Children's Hospital of Fudan University, National Children's Medical Center, Shanghai, China; ${ }^{5}$ Department of Pediatrics, Xiamen Children's Hospital, Xiamen, China; ${ }^{6}$ Department of Child Health Care, Children's Hospital, Zhejiang University School of Medicine, Hangzhou, China

Contributions: (I) Conception and design: T Xiao, B Wu, Z Zhao, H Wang, W Zhou; (II) Administrative support: Z Zhao, H Wang, W Zhou; (III) Provision of study materials or patients: T Xiao, B Wu, Y Cao, R Liu, G Cheng, L Wang, D Zhuang; (IV) Collection and assembly of data: T Xiao, B Wu, R Liu; (V) Data analysis and interpretation: T Xiao, B Wu, R Liu; (VI) Manuscript writing: All authors; (VII) Final approval of manuscript: All authors.

\#These authors contributed equally to this work.

Correspondence to: Zhengyan Zhao. Department of Child Health Care, Children's Hospital, Zhejiang University School of Medicine, Hangzhou 310058, China. Email: zhaozy@zju.edu.cn; Huijun Wang. Center for Molecular Medicine, Children's Hospital of Fudan University, National Children's Medical Center, 399 Wanyuan Road, Shanghai 201102, China. Email: huijunwang@fudan.edu.cn; Wenhao Zhou. Department of Neonatology, Children's Hospital of Fudan University, National Children's Medical Center; Center for Molecular Medicine, Children's Hospital of Fudan University, National Children’s Medical Center, 399 Wanyuan Road, Shanghai 201102, China. Email: zhouwenhao@fudan.edu.cn.

Background: An elevated level of creatine kinase $(\mathrm{CK})$ is usually the primary screening marker for Duchenne muscular dystrophy (DMD)/Becker muscular dystrophy (BMD). This study investigated the clinical application of next-generation sequencing (NGS) in newborns with a possible diagnosis of DMD/ $\mathrm{BMD}$ in the neonatal intensive care unit (NICU).

Methods: NGS data from the NICU between June 1, 2016, and June 30, 2020, were reanalyzed by an in-house pipeline. Other methods confirmed the genetic findings, and clinical follow-up was performed until August 1, 2020.

Results: Of the 10,481 newborns, $19(0.18 \%, 19 / 10,481)$ cases with pathogenic variations of the $D M D$ gene were identified, including 13 (68.4\%, 13/19) deletions, 4 (21.1\%, 4/19) duplications, and 2 (10.5\%, 2/19) nonsense mutations. Eight of the cases were diagnosed with DMD. Therapeutic strategies were modified for these patients. Six cases were diagnosed with BMD. Five patients except for 1 deceased patient were further followed-up, and clinical management was adjusted based on the clinical symptoms. The remaining 5 cases were indeterminate for DMD and BMD. Genetic counseling and further follow-up were performed or suggested.

Conclusions: Our study showed that DMD/BMD could be diagnosed earlier in the neonatal stage before the typical clinical symptoms appear. Early diagnosis may provide an opportunity for guiding the care and treatment of patients. However, ethical issues need to be kept in mind in the process of genetic counseling.

Keywords: Duchenne muscular dystrophy (DMD); Becker muscular dystrophy (BMD); next-generation sequencing (NGS)

Submitted Oct 25, 2020. Accepted for publication Feb 07, 2021.

doi: $10.21037 /$ atm-20-7102

View this article at: http://dx.doi.org/10.21037/atm-20-7102

(c) Annals of Translational Medicine. All rights reserved. 


\section{Introduction}

Duchenne muscular dystrophy (DMD) and its less severe form Becker muscular dystrophy (BMD) are inherited progressive myopathic diseases resulting from mutations in the $D M D$ gene located on the $\mathrm{X}$ chromosome. This gene spans approximately $2.4 \mathrm{Mb}$ of genomic DNA, including 79 exons and 78 introns, and affects skeletal and myocardial muscle and the brain's development. At present, the overall incidence is estimated to be 1 case per 5,000 live male births according to 10 large DMD newborn screening programs (1). DMD patients are characterized by progressive muscular damage and degeneration, resulting in mildly delayed motor milestones, muscular weakness, loss of ambulation, cardiomyopathy, and cardiac or respiratory failure. Also, some patients can present cognitive dysfunction (2). In contrast, BMD patients present a later and slower progression of muscular damage. The severity of phenotypes is related, at least in part, to whether the amino acid translational reading frame is disrupted or preserved by the deletion/duplication mutations (3).

In practice, patients with $\mathrm{DMD} / \mathrm{BMD}$ are usually not symptomatic until 2 to 5 years old. The typical diagnostic process usually begins in early childhood after the characteristic clinical symptoms are noticed (1). Further investigations, including metabolic tests, genetic tests, and possible muscle biopsy, are performed (1). Therefore, a delay of up to 2 years between the first onset of symptoms and the diagnosis is common in affected patients (4). To date, many studies have suggested that earlier intervention, such as glucocorticoid treatment $(5,6)$, multidisciplinary care (7), and better family planning (8), could improve neuromuscular function and quality of life and extend the lifespan of DMD patients (9). Furthermore, molecular and gene therapies for DMD have been on the horizon (10). Therefore, a timely and accurate diagnosis of DMD/BMD is crucial for management.

Currently, population-based DMD newborn screening is based on creatine kinase (CK) from dried blood spots. An elevated CK level is the primary screening marker and assists in identifying patients with suspected myopathic diseases. However, its performance has not been satisfactory under clinical conditions since CK levels can be elevated due to birth trauma, asphyxia, and infection, which produces false positives in sick neonates (8). With the widescale implementation of next-generation sequencing (NGS) in recent years, the application of NGS provides exciting possibilities in the field of critical care medicine (11-13). A previous study indicated that neonatal intensive care unit (NICU) patients were at high risk of genetic conditions, and many trials have been launched to investigate genetic conditions in the NICU setting, which could help to identify the target population (14). Therefore, our study aims to perform genetic identification of pathogenic $D M D$ gene variations in newborns from the NICU using clinical exome sequencing data and investigate the impact of the early identification of pathogenic variations of the $D M D$ gene on the clinical decision. We present the following article in accordance with the MDAR and STROBE reporting checklist (available at http://dx.doi.org/10.21037/ atm-20-7102).

\section{Methods}

\section{Study subjects}

The clinical exome sequencing data of 10,486 individuals admitted to the NICU at Fudan University of Children from June 1, 2016, to June 30, 2020, were reanalyzed by an inhouse pipeline for genetic analysis. The inclusion criteria were as follows: (I) patients were from the NICU; (II) postnatal age was less than 28 days; (III) biological parents or guardians provided informed consent. Clinical data were extracted from medical records, including clinical features, laboratory results, and follow-up information in the clinic. Patients were excluded if clinical information was absent. A molecular geneticist and a neurologist evaluated genotype and phenotype correlations for DMD. The last follow-up was performed by phone call. The study was conducted following the Declaration of Helsinki (as revised in 2013). The Children's Hospital of Fudan University ethics committee approved this study since the study began (2020-441). Written informed consent was obtained from all participants.

\section{Data analysis}

The raw data of FastQ was reanalyzed using the in-house analytical pipeline (15) (Figure S1). GATK best practice was performed for single nucleotide variants $(\mathrm{SNVs}) / \mathrm{small}$ indels for variant calling. Copy number variants $(\mathrm{CNVs})$ were detected by HMZDelFinder (16) and CANOES (17) separately, and the results were merged. HMZDelFinder is an algorithm for the detection of rare and intragenic homozygous and hemizygous (HMZ) deletions. BAM files and VCF files of individuals were extracted as the input for variant detection. The application was conducted 
under default-set thresholds. In the published version of CANOES (17), researchers stated that all CNV callings were restricted to the autosomes due to complications resulting from the samples being of different genders. We extended the $\mathrm{CNV}$ calling to chromosome $\mathrm{X}$ by adjusting the target region coverage according to the samples' gender. The detailed extension process included adjusting the $\mathrm{X}$ chromosome coverage according to gender and evaluating the baseline for $\mathrm{CNV}$ detection on the $\mathrm{X}$ chromosome using autosome and $\mathrm{X}$ chromosome coverage data. Both read coverage and the GC content of the exons were used to call CNVs by CANOES. The annotation and filtrations of both SNVs and CNVs have been described in published work (15). SNVs were then validated by Sanger sequencing, while diagnostic CNVs were confirmed by multiplex ligation-dependent probe amplification (MLPA).

The human genome variation society (HGVS) nomenclature and the predicted mutation type of $D M D$ gene variations were made about the Leiden muscular dystrophy database website (http://www.dmd.nl/). The previously reported cases were extracted from the Leiden muscular dystrophy database (18), the UMD-DMD database (19), the eDystrophin database, the TREAT-NMD DMD Global database (20), and the human gene mutation database (HGMD).

\section{Results}

\section{Genetic analysis identified 19 newborns with DMD gene pathogenic variations}

The genetic data from 10,481 patients from the NICU between June 1, 2016, and June 30, 2020, were reanalyzed. Nineteen $(0.18 \%, 19 / 10,481)$ newborns with pathogenic variations in the $D M D$ gene were identified by the in-house pipeline, including $17 \mathrm{CNVs}(89.5 \%, 17 / 19)$ and $2 \mathrm{SNVs}$ (10.5\%, 2/19). Among the $17 \mathrm{CNVs}, 76.5 \%$ (13/17) were deletions and $23.5 \%$ (4/17) were duplications (Figure 1). Eight infants were clinically diagnosed with DMD. Six infants were clinically diagnosed with $\mathrm{BMD}$, and 1 of them died during the neonatal period. The remaining 5 infants had indeterminate DMD or BMD, and 1 of these 5 infants died during the neonatal period. The results of the MLPA and Sanger sequencing are described in the supplementary file (Figure S2).

\section{Clinical diagnosis of 19 newborns with DMD gene pathogenic variations}

The genotype and phenotype correlation, the genetic evidence, and clinical assessment at last follow-up for the 19 newborns with $D M D$ gene pathogenic variations are summarized in Table 1. Except for 2 cases (Neo_18, Neo_19) with nonsense mutations, 7 cases were outof-frame deletions, 1 case (Neo_9) was an out-of-frame duplication, 6 cases were in-frame deletions, 1 case (Neo_7) was an in-frame duplication, and the remaining 2 cases (Neo_1, Neo_17) were difficult to predict, with $D M D$ gene exon 1-7 duplications.

Eight patients (No. 1-8) were diagnosed with DMD according to the out-of-frame mutation type and the mutation hotspots of previous DMD patients. Three newborns (Neo_11, Neo_14, Neo_16) had a family history. The variant of Neo_9 was a novel pathogenic variation, but the predicted mutation type was an out-offrame duplication, and he had a CK level of 50,000 IU/L. Therefore, he was classified in the group of DMD patients.

Six newborns (No. 9-14) were diagnosed with BMD based on in-frame mutation type and the mutation hotspots of previous BMD patients. No muscular biopsy has been performed on these 6 patients.

Five newborns (No. 15-19) could not be distinguished between DMD and BMD based on limited phenotypes and $D M D$ gene pathogenic variations. Neo_1 and Neo_17 had exon 1-7 duplication, which makes it difficult to predict mutation type. In a previous report (29), a patient who harbored exon 1-7 duplication was diagnosed with DMD. However, due to this area's phenotype variability, it is hard to predict the mutation type of our patients. Clinical followup and genetic consultation were recommended. Neo_7 (with duplication of exon 1-2) and 2 cases with nonsense mutations (Neo_18 and Neo_19) had limited phenotypes. Also, there was insufficient evidence of the correlation between phenotype and the variations of these 3 infants. A muscluar biopsy has not been performed on these 4 survival patients.

\section{Clinical assessment and follow-up of the 19 patients}

Among the 19 newborns with $D M D$ gene pathogenic variations, 8 infants aged from 9 months to 3 years were diagnosed with DMD. They were classified as stage 1 based on the updated 2010 DMD care considerations supported by the US Centers for Disease Control and Prevention (CDC) (Figure 2). These 8 patients (No. 1-8) were referred to the DMD clinic for scheduled multidisciplinary followup and treatment guidance.

Six patients (No. 9-14) aged from 9 months to 3 years 


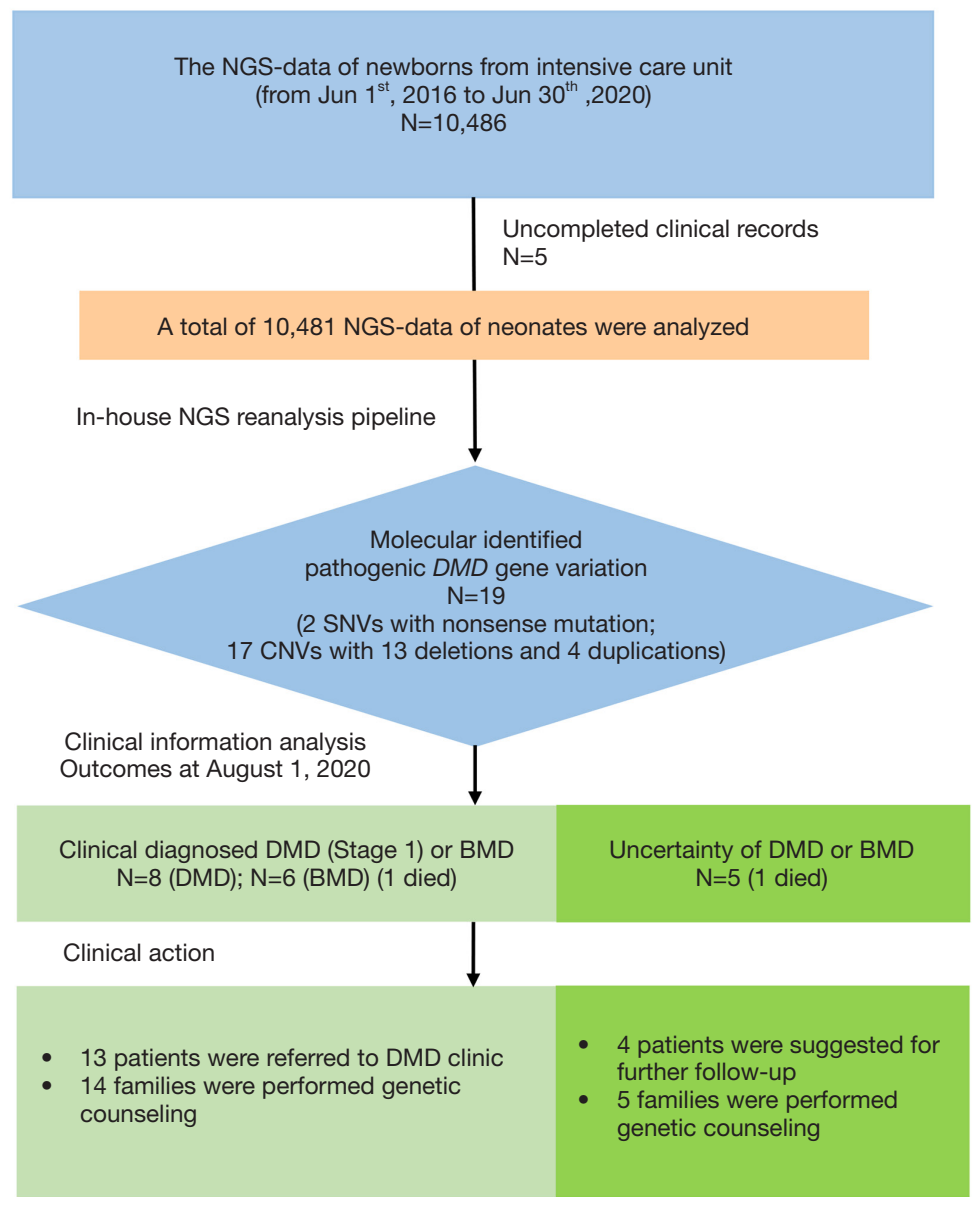

Figure 1 The study algorithm of the genetic identification of DMD gene pathogenic variations via next-generation sequencing. NGS, nextgeneration sequencing; BMD, Becker muscular dystrophy; DMD, Duchenne muscular dystrophy.

were diagnosed with BMD. We could not conclude the severity of BMD based on the limited phenotypes. Therefore, 5 patients except for 1 deceased infant (Neo_6) were referred to the DMD clinic for further follow-up, and the management plans were based on clinical symptoms. Genetic counseling was offered to all the families of patients with BMD.

For the remaining 5 (No. 15-19) newborns without a definitive diagnosis of DMD or BMD, 4 of them except for 1 deceased infant (Neo_17) were suggested to undergo further follow-up and investigation in a genetic clinic. Five of the families underwent genetic counseling. However, none of the 4 patients underwent a muscular biopsy.

Neo_5, born at the gestational age of 36 weeks, was admitted due to suspected sepsis with an incidental finding of elevated CK. His electrocardiogram showed sinus rhythm and $\mathrm{T}$ wave change. No family history was reviewed. Because of persistent elevated CK, he was referred to the neurological specialty clinic for follow-up since the neonatal period. At 3 months of age, he presented the calf's pseudohypertrophy on examination, and CK was persistently elevated. Combined with the earlier molecular results of out-of-frame deletions of exons 45-50 in the $D M D$ gene, he had a timely clinical diagnosis of DMD. The physician discussed the management plan with the family and planned to administer corticosteroids at 4 years old. Currently, he is 2 years old with no motor or cardiac dysfunction.

Neo_16 presented with hyperbilirubinemia, mild hypotonia, and elevated CK at 3 days of life. His brother presented difficulty in walking up steps and was diagnosed with DMD at 7 years old. Neo_16 was diagnosed with DMD at 1 month of age due to persistently elevated CK and identification of out-of-frame deletions of exons $22-37$ in 


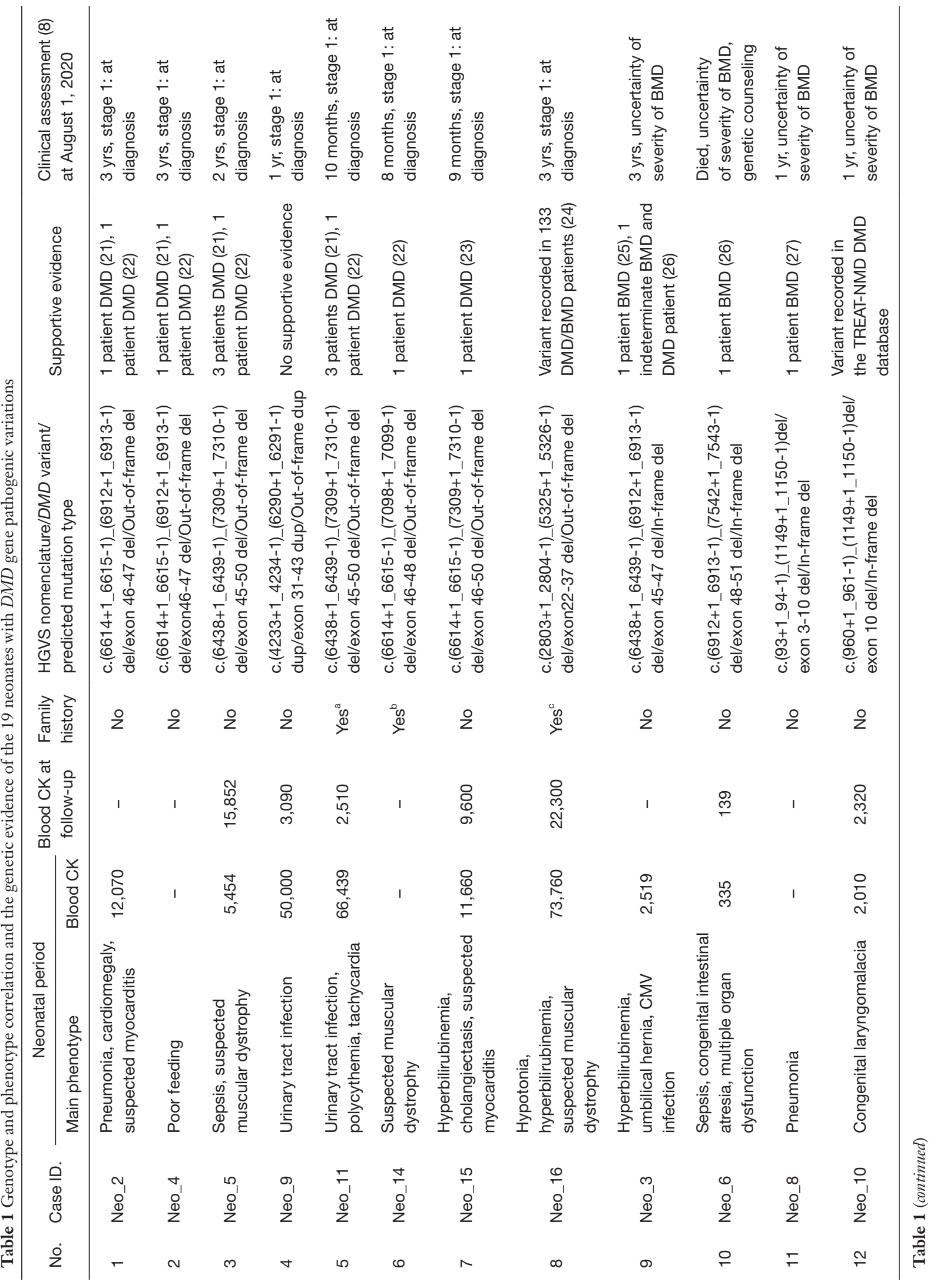




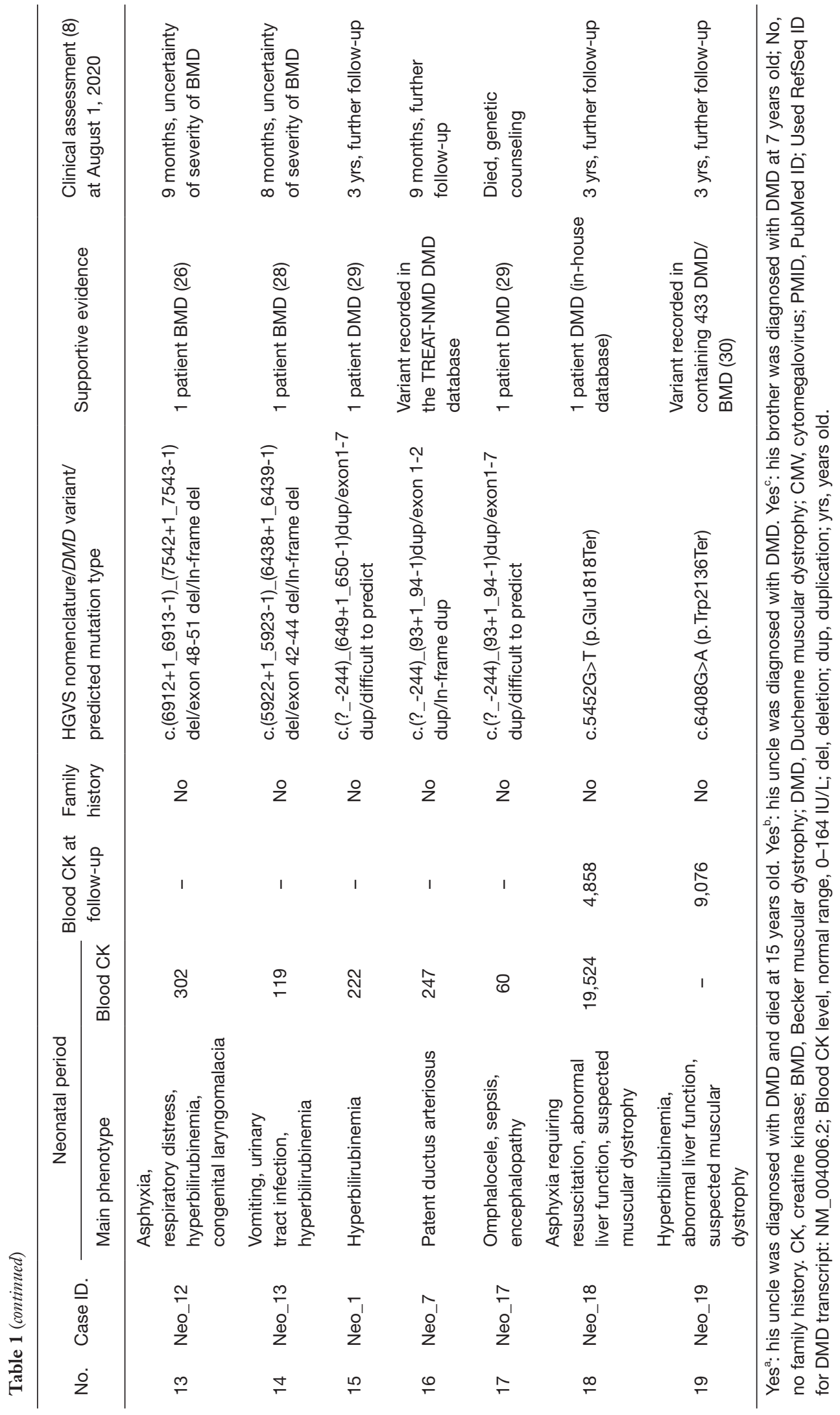




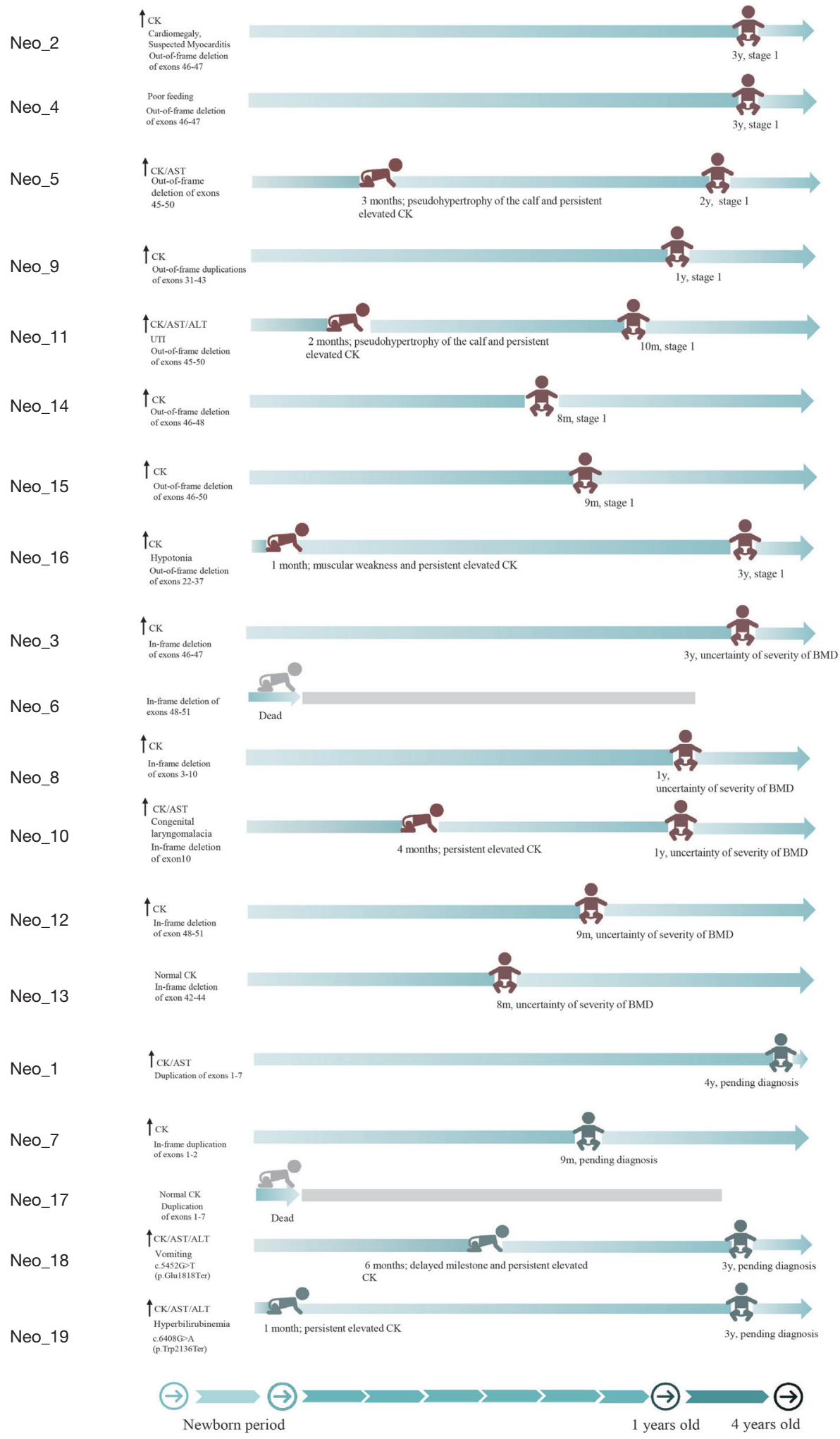

Figure 2 The clinical history of 19 patients with $D M D$ gene pathogenic variations. CK, serum creatine kinase; AST, aspartate transaminase; ALT, aspartate aminotransferase; CK, creatine kinase; BMD, Becker muscular dystrophy. 
the $D M D$ gene. Multidisciplinary care was implemented, and family support was offered at 1 month of age. At present, he is 3 years old, and no abnormalities in motor and cardiac function have been found.

Neo_18 was admitted due to asphyxia, required resuscitation, and had an abnormal liver function at 2 days of life. He had an incidental finding of elevated CK. The elevated CK was persistent, ranging from 2,028 to 19,524 $\mathrm{UI} / \mathrm{L}$, and 1 nonsense variation in the $D M D$ (c.5452G>T, p.Glu1818Ter) gene was detected. During the scheduled clinical assessment, he could not sit alone at 6 months of life, indicating delayed milestones. Family education has been provided. At present, he is 3 years old without motor dysfunction.

\section{Discussion}

To our knowledge, genetic testing can provide a confirmed diagnosis of dystrophinopathy without typical findings from muscle biopsy in most suspected cases (9). The current recommendation suggests that muscle biopsy is optional to offer to patients according to the clinical situation and differential diagnosis of other muscular dystrophy types (9). If the genetic test confirms the diagnosis of DMD, a muscle biopsy is not necessary. The recent DMD care considerations guide assessments and interventions based on disease stages (8) and suggest that clinical care coordination is crucial in DMD management. These strategies include neuromuscular, rehabilitation, endocrine (growth, puberty, and adrenal insufficiency), gastrointestinal (including nutrition and dysphagia), respiratory, cardiac, bone health, and orthopedic management (8). Evidence has revealed that targeted intervention for known clinical symptoms can change the natural history of DMD and help patients achieve a longer lifespan (9). Glucocorticoids are the only therapeutic medication for DMD patients aged 4 years or older $(8,31)$. Studies have demonstrated that glucocorticoids can improve motor function and pulmonary function, delay ambulation loss, and extend the lifespan (31). With the widespread understanding of dystrophinopathy at the molecular level and the side-effects of glucocorticoids (8), new therapeutic medications are emerging. A study indicated that new medications, including eteplirsen, golodirsen, and ataluren, have been approved by the FDA, and ataluren can be administered as early as 2 years old (4).

Our study identified 19 newborns with $D M D$ gene pathogenic variations from 10,481 NICU patients, and the incidence of $D M D$ gene pathogenic variations was $0.18 \%$
$(19 / 10,481)$ in our cohort. With early genetic findings of $D M D$ gene pathogenic variations, all the infants were closely monitored at an early age. The early identification of $D M D$ gene pathogenic variations in addition to $\mathrm{CK}$ levels avoided muscle biopsy, altered the clinical decision, and guided physicians to decide the best practices. Among these newborns, Neo_16, with a family history of DMD, presented with hypotonia and persistently elevated CK in the neonatal period, which indicates that DMD-relevant phenotypes can manifest as early as the neonatal period. A similar finding from a case report showed that an infant born at 31 weeks presented with hypotonia since birth and was diagnosed with DMD and X-linked myotubular myopathy at around 1 month of age (32).

However, 5 newborns with $D M D$ gene pathogenic variations in our study did not have a definitive diagnosis of DMD or BMD because of limited phenotypes and nonhotspot mutation types. There was limited evidence of an association between phenotypic expression and the variations of these 5 newborns. Therefore, physicians referred them, except for 1 deceased infant, to the genetic consulting clinic for further follow-up. These 4 infants may require further evaluation of CK levels or muscle biopsy depending on the further clinical situation (9). Moreover, studies have indicated that some patients with $D M D$ gene mutations could present with dilated cardiomyopathy and mild cognitive impairment or global developmental delay $(3,9)$. Therefore, these genetic findings can help physicians recognize the relevant but nonspecific phenotypes early and potentially avoid a delayed diagnosis of DMD/BMD. The MDSTARnet cohort study indicated that earlier age of onset of first symptoms was a risk factor for more rapid progression of muscle weakness (33). Thus, early identification of the relevant phenotype could also help to predict the prognosis of patients with DMD/BMD.

Importantly, we faced ethical issues during the re-analysis of these data. Eight infants with DMD at stage 1 of the disease and 6 patients with BMD were pre-symptomatic. They will develop muscular weakness in later life, and there is no curative treatment. Therefore, these infants may experience social stigmatization of persons with disabilities and feel negatively in their later life. For the 2 deceased newborns with $D M D$ gene pathogenic variations, their families may feel negatively about having another baby. For the 5 newborns without a definitive diagnosis of DMD or $\mathrm{BMD}$, potential harm or anxiety to families may have been caused by the lack of closure.

On the other hand, 2 studies in a small number of 
participants revealed that more than $90 \%$ of parents of affected children and expectant parents supported the DMD newborn screening program $(34,35)$. Also, our study indicated that the NGS identification of $D M D$ gene pathogenic variations in the NICU did impact future clinical management for these patients. Therefore, with the more widespread realization of the benefits of NGS identification of $D M D$ gene pathogenic variations in practice, along with muscle-directed therapies and organized multidisciplinary care, we may have a greater ability to deal with the ethical issues.

Our study has several limitations. First, analysis of the clinical exome sequencing data used for DMD/BMD was performed on patients from a single hospital; therefore the generalizability may be limited. Second, the CK levels' dynamic changes were absent because patients did not present for follow-up in the clinic consistently. The last clinical follow-up was via a telephone call. Therefore, the clinical symptoms provided by parents may not be accurate. Third, our study ended on August 1, 2020. Most of the patients were less than 4 years old. Therefore, it will be essential to continue tracking these families to assess longterm benefits. Additionally, given the limited long-term neuromuscular assessments and the absence of muscle biopsy, we could not confirm DMD or BMD diagnosis in 5 cases. Lastly, for 6 patients with a BMD diagnosis, further management should be modified based on the severity of the disease.

In conclusion, with the early identification of $D M D$ gene pathogenic variations, in addition to CK levels and family history, we can diagnose patients with DMD in the neonatal period before the onset of typical clinical symptoms, and further multidisciplinary management and follow-up can be implemented at an early age for these affected children. This strategy for DMD/BMD diagnosis can improve the outcomes of affected patients, but further clinical trials are urgently required. Notably, for patients with incomplete clinical data and non-hotspots variations, physicians must balance the infant and parents' best interests when they consult with families.

\section{Acknowledgments}

The authors sincerely thank all the family members for their participation in this study. We thank Prof. Xihua Li (molecular geneticist) and Dr. Yuanfeng Zhou (neurologist) in our hospital for their professional guidance on genotype and phenotype analysis.
Funding: This study was supported by Shanghai Municipal Science and Technology Major Project (Grant No. 2017SHZDZX01) and Shanghai's Science and Technology Commission (20Z11900600).

\section{Footnote}

Reporting Checklist: The authors have completed the MDAR and STROBE reporting checklist. Available at http://dx.doi. org/10.21037/atm-20-7102

Data Sharing Statement: Available at http://dx.doi. org/10.21037/atm-20-7102

Peer Review File: Available at http://dx.doi.org/10.21037/ atm-20-7102

Conflicts of Interest: All authors have completed the ICMJE uniform disclosure form (available at http://dx.doi. org/10.21037/atm-20-7102). The authors have no conflicts of interest to declare.

Ethical Statement: The authors are accountable for all aspects of the work in ensuring that questions related to the accuracy or integrity of any part of the work are appropriately investigated and resolved. The study was conducted per the Declaration of Helsinki (as revised in 2013). The Children's Hospital of Fudan University ethics committee approved this study since the study began (2020441). Written informed consent was obtained from all participants.

Open Access Statement: This is an Open Access article distributed in accordance with the Creative Commons Attribution-NonCommercial-NoDerivs 4.0 International License (CC BY-NC-ND 4.0), which permits the noncommercial replication and distribution of the article with the strict proviso that no changes or edits are made and the original work is properly cited (including links to both the formal publication through the relevant DOI and the license). See: https://creativecommons.org/licenses/by-nc-nd/4.0/.

\section{References}

1. Gatheridge MA, Kwon JM, Mendell JM, et al. Identifying Non-Duchenne Muscular Dystrophy-Positive and False Negative Results in Prior Duchenne Muscular Dystrophy Newborn Screening Programs: A Review. JAMA Neurol 
2016;73:111-6.

2. Darras BT, Urion DK, Partha GS. Dystrophinopathies. GeneReviews. Available online: www.ncbi.nlm.nih.gov/ books/NBK1119/

3. Muntoni F, Torelli S, Ferlini A. Dystrophin and mutations: one gene, several proteins, multiple phenotypes. Lancet Neurol 2003;2:731-40.

4. Vita GL, Vita G. Is it the right time for an infant screening for Duchenne muscular dystrophy? Neurol Sci 2020;41:1677-83.

5. Schram G, Fournier A, Leduc H, et al. All-Cause Mortality and Cardiovascular Outcomes With Prophylactic Steroid Therapy in Duchenne Muscular Dystrophy. J Am Coll Cardiol 2013;61:948-54.

6. McDonald CM, Henricson EK, Abresch RT, et al. Long-term effects of glucocorticoids on function, quality of life, and survival in patients with Duchenne muscular dystrophy: a prospective cohort study. Lancet 2018;391:451-61.

7. Passamano L, Taglia A, Palladino A, et al. Improvement of survival in Duchenne Muscular Dystrophy: retrospective analysis of 835 patients. Acta Myol 2012;31:121-5.

8. Birnkrant DJ, Bushby K, Bann CM. Diagnosis and management of Duchenne muscular dystrophy, part 1: diagnosis, and neuromuscular, rehabilitation, endocrine, and gastrointestinal and nutritional management. Lancet Neurol 2018;17:251-67.

9. Bushby K, Finkel R, Birnkrant DJ, et al. Diagnosis and management of Duchenne muscular dystrophy, part 1: diagnosis, and pharmacological and psychosocial management. Lancet Neurol 2010;9:77-93.

10. Muntoni F, Wells D. Genetic treatments in muscular dystrophies. Curr Opin Neurol 2007;20:590-4.

11. Bodian DL, Klein E, Iyer RK, et al. Utility of wholegenome sequencing for detection of newborn screening disorders in a population cohort of 1,696 neonates. Genet Med 2016;18:221-30.

12. Bassaganyas L, Freedman G, Vaka D, et al. Whole exome and whole genome sequencing with dried blood spot DNA without whole genome amplification. Hum Mutat 2018;39:167-71.

13. Adhikari AN, Gallagher RC, Wang Y, et al. The role of exome sequencing in newborn screening for inborn errors of metabolism. Nat Med 2020;26:1392-7.

14. Swaggart KA, Swarr DT, Tolusso LK, et al. Making a Genetic Diagnosis in a Level IV Neonatal Intensive Care Unit Population: Who, When, How, and at What Cost? J Pediatr 2019;213:211-7.e4.
15. Dong X, Liu B, Yang L, et al. Clinical exome sequencing as the first-tier test for diagnosing developmental disorders covering both CNV and SNV: a Chinese cohort. J Med Genet 2020;57:558-66.

16. Gambin T, Akdemir ZC, Yuan B, et al. Homozygous and hemizygous CNV detection from exome sequencing data in a Mendelian disease cohort. Nucleic Acids Res 2017;45:1633-48.

17. Backenroth D, Homsy J, Murillo LR, et al. CANOES: detecting rare copy number variants from whole exome sequencing data. Nucleic Acids Res 2014;42:e97.

18. Aartsma-Rus A, Van Deutekom JCT, Fokkema IF, et al. Entries in the Leiden Duchenne muscular dystrophy mutation database: An overview of mutation types and paradoxical cases that confirm the reading-frame rule. Muscle Nerve 2006;34:135-44.

19. Tuffery-Giraud S, Beroud C, Leturcq F, et al. Genotype-phenotype analysis in 2,405 patients with a dystrophinopathy using the UMD-DMD database: a model of nationwide knowledgebase. Hum Mutat 2009;30:934-45.

20. Bladen CL, Salgado D, Monges S, et al. The TREATNMD DMD Global Database: analysis of more than 7,000 Duchenne muscular dystrophy mutations. Hum Mutat 2015;36:395-402.

21. Dent KM, Dunn DM, von Niederhausern AC, et al. Improved molecular diagnosis of dystrophinopathies in an unselected clinical cohort. Am J Med Genet A 2005;134:295-8.

22. Moizard MP, Billard C, Toutain A, et al. Are Dp71 and Dp140 brain dystrophin isoforms related to cognitive impairment in Duchenne muscular dystrophy? Am J Med Genet 1998;80:32-41.

23. Ulgenalp A, Oner FH, Soylev MF, et al. Electroretinographic findings in Duchenne/Becker muscular dystrophy and correlation with genotype. Ophthalmic Genet 2002;23:157-65.

24. Lalic T, Vossen RH, Coffa J, et al. Deletion and duplication screening in the DMD gene using MLPA. Eur J Hum Genet 2005;13:1231-4.

25. Tay SK, Khng HH, Low PS, et al. Diagnostic strategy for the detection of dystrophin gene mutations in asian patients and carriers using immortalized cell lines. J Child Neurol 2006;21:150-5.

26. Beggs AH, Hoffman EP, Snyder JR, et al. Exploring the molecular basis for variability among patients with Becker muscular dystrophy: dystrophin gene and protein studies. Am J Hum Genet 1991;49:54-67. 
27. Kaspar RW, Allen HD, Ray WC, et al. Analysis of dystrophin deletion mutations predicts age of cardiomyopathy onset in becker muscular dystrophy. Circ Cardiovasc Genet 2009;2:544-51.

28. Morandi L, Mora M, Confalonieri V, et al. Dystrophin characterization in BMD patients: correlation of abnormal protein with clinical phenotype. J Neurol Sci 1995;132:146-55.

29. Mah JK, Selby K, Campbell C, et al. A population-based study of dystrophin mutations in Canada. Can J Neurol Sci 2011;38:465-74.

30. Bai Y, Li S, Zong YN, et al. Mutation screening of 433 families with Duchenne/Becker muscular dystrophy. Zhonghua Yi Xue Za Zhi 2016;96:1261-9.

31. Matthews E, Brassington R, Kuntzer T, et al. Corticosteroids for the treatment of Duchenne muscular dystrophy. Cochrane Database Syst Rev

Cite this article as: Xiao T, Wu B, Cao Y, Liu R, Cheng G, Wang L, Zhuang D, Zhao Z, Wang H, Zhou W. Genetic identification of pathogenic variations of the $D M D$ gene: a retrospective study from 10,481 neonatal patients based on next-generation sequencing data. Ann Transl Med 2021;9(9):766. doi: 10.21037/atm-20-7102
2016;(5):CD003725.

32. Varma U, Mukherjee D, Hughes I, et al. X-Linked Myotubular Myopathy and Duchenne Muscular Dystrophy in a Preterm Infant: A Rare Combination. Pediatrics 2020;146:e20182879.

33. Ciafaloni E, Kumar A, Liu K, et al. Age at onset of first signs or symptoms predicts age at loss of ambulation in Duchenne and Becker Muscular Dystrophy: Data from the MD STARnet. J Pediatr Rehabil Med 2016;9:5-11.

34. Chung J, Smith AL, Hughes SC, et al. Twenty-year follow-up of newborn screening for patients with muscular dystrophy. Muscle Nerve 2016;53:570-8.

35. Wood MF, Hughes SC, Hache LP, et al. Parental attitudes toward newborn screening for Duchenne/Becker muscular dystrophy and spinal muscular atrophy. Muscle Nerve 2014;49:822-8. 


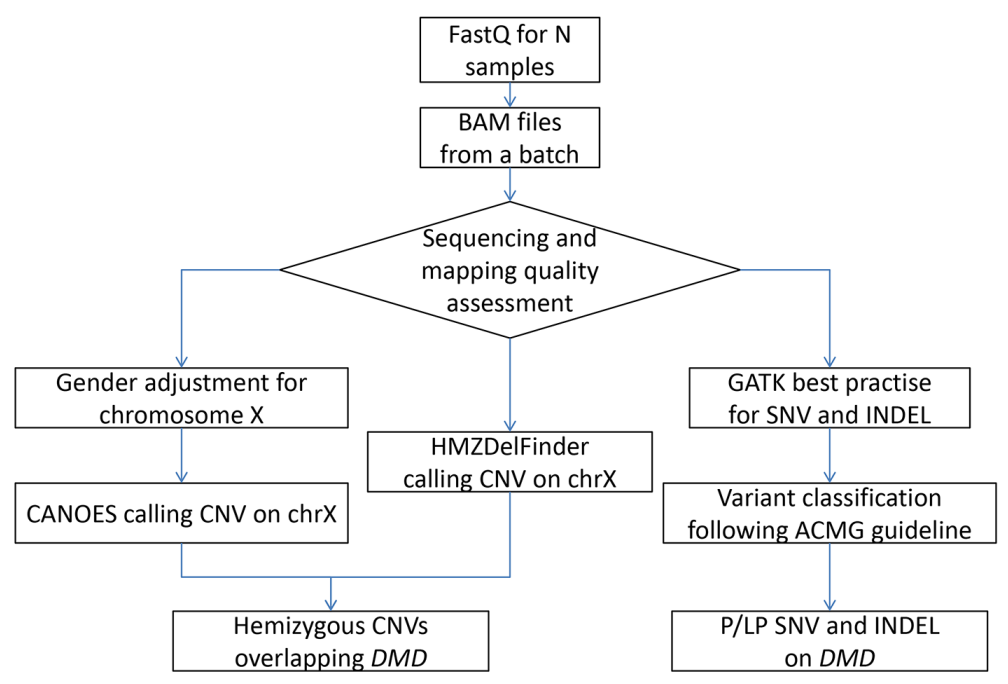

Figure S1 In-house analytical pipeline.

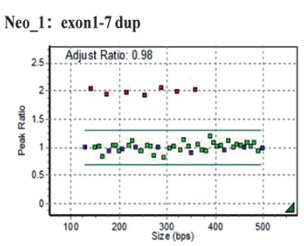

Neo_2: exon 46-47 del

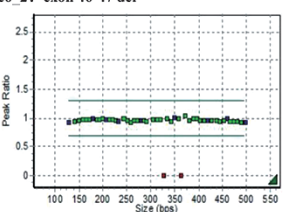

Neo_3: exon 45-47 del

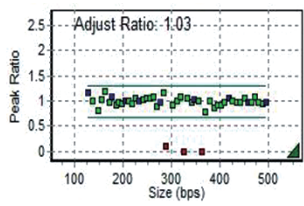

Neo_4: exon46-47 del

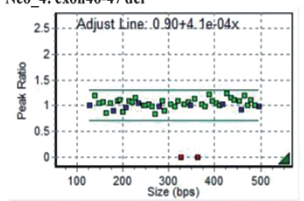

Neo_5: exon 45-50 del

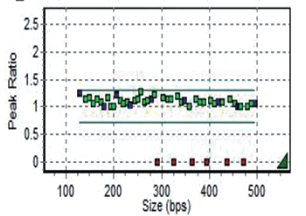

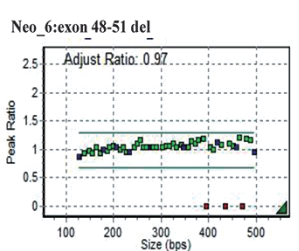

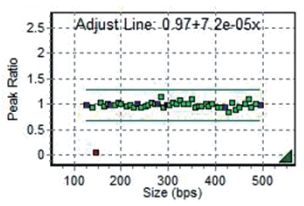

Neo_7: exon 1-2 dup

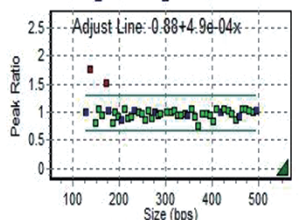

Neo_ 8: exon 3-10 del
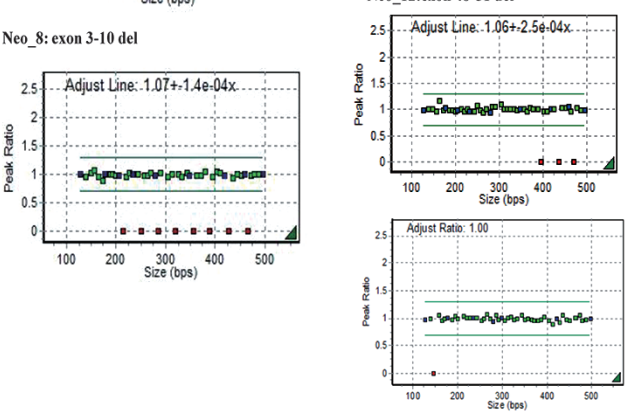
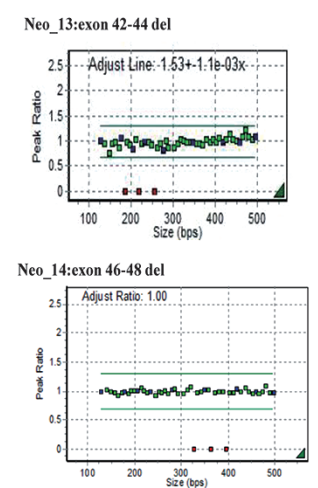

Neo_ 15 :exon 46-50 del

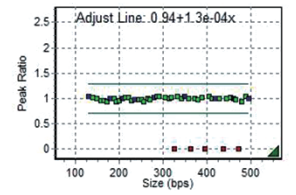

Neo_16: exon22-37 del
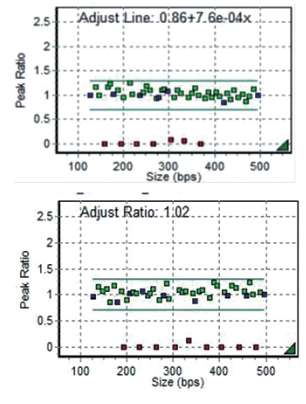

Neo_17:exon1-7 dup

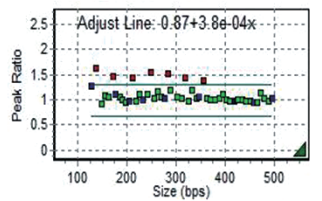

Neo_18:c.5452G>T (p.Glu1818Ter)

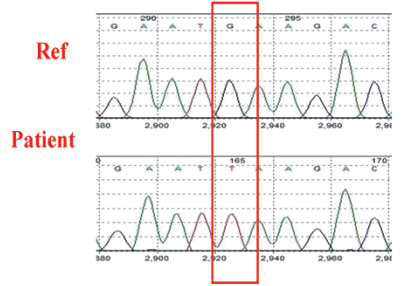

Neo_19:c.6408G>A(p.Trp2136Ter)

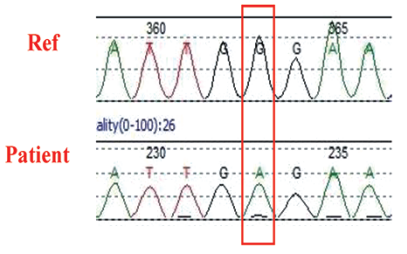

Figure S2 The results of Sanger sequencing and multiplex ligation-dependent probe amplification. 